\title{
World-GAN: a Generative Model for Minecraft Worlds
}

\author{
Maren Awiszus* \\ Institut für Informationsverarbeitung \\ Leibniz University Hannover \\ Hannover, Germany \\ awiszus@tnt.uni-hannover.de
}

\author{
Frederik Schubert* \\ Institut für Informationsverarbeitung \\ Leibniz University Hannover \\ Hannover, Germany \\ schubert@tnt.uni-hannover.de
}

\author{
Bodo Rosenhahn \\ Institut für Informationsverarbeitung \\ Leibniz University Hannover \\ Hannover, Germany \\ rosenhahn@tnt.uni-hannover.de
}

\begin{abstract}
This work introduces World-GAN, the first method to perform data-driven Procedural Content Generation via Machine Learning in Minecraft from a single example. Based on a 3D Generative Adversarial Network (GAN) architecture, we are able to create arbitrarily sized world snippets from a given sample. We evaluate our approach on creations from the community as well as structures generated with the Minecraft World Generator. Our method is motivated by the dense representations used in Natural Language Processing (NLP) introduced with word2vec [1]. The proposed block2vec representations make World-GAN independent from the number of different blocks, which can vary a lot in Minecraft, and enable the generation of larger levels. Finally, we demonstrate that changing this new representation space allows us to change the generated style of an already trained generator. World-GAN enables its users to generate Minecraft worlds based on parts of their creations.
\end{abstract}

Index Terms-Minecraft, Level, Generation, PCG, GAN, SinGAN, Single Example, Scales, Style, Representation

\section{INTRODUCTION}

Procedural Content Generation (PCG) has been applied to many different areas and games [2], [3]. Recently, the progress in Machine Learning has especially spurred research in the field of PCG via Machine Learning (PCGML) [4]. While there are methods to generate levels for $2 \mathrm{D}$ games such as Super Mario Bros. [5], generating levels in 3D is an open area of research. There has been work on generating levels for the 3D game Doom [6], but the generation process relies on generating $2 \mathrm{D}$ descriptions of the level layout that prohibit complex vertical structures.

There are 3D games that use grammars and rule-based algorithms for PCG in level generation. However, these games do not yet use Machine Learning in their generation process and are thus only extendable by programming new rules. The most prominent game in this domain is Minecraft [7], which can generate endless worlds in a 3D voxel space. Minecraft's World Generator is intricately handcrafted to generate vast landscapes, filled with structures like villages or caves with mine shafts. These landscapes also change with so-called biomes, which define the type of area, from plains to deserts to beaches. Human generated content also plays an important role in Minecraft, but the structures have to be placed manually

*Equal contribution

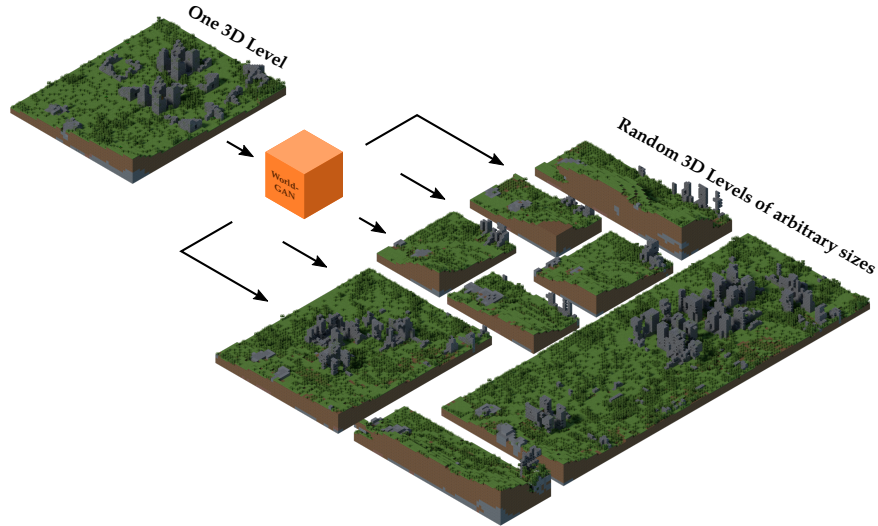

Fig. 1: Examples of generated Minecraft world snippets trained on a sample of handcrafted stone ruins in a field of grass. The samples can be generated in arbitrary sizes and capture the structures of the ruins and the terrain of the original area.

in a fixed world and the World Generator cannot learn to reconstruct or generate newly built structures on its own.

In this paper, we bridge the gap between the rule-based PCG of the Minecraft World Generator and custom structures that were designed by hand, as our proposed method aims to learn and generate structures directly in 3D voxel space (compare Fig. 1. all world visualizations are made using Mineways [8] and Blender [9]).

In summary, our contributions are:

- We introduce a 3D Generative Adversarial Network (GAN) architecture for level generation in Minecraft.

- Our proposed dense token representations using block2vec enable the processing of larger world snippets.

- Editing this representation space allows the application of style changes to generated levels without further training.

- Using a current version of Minecraft (1.16) makes our method widely applicable for practitioners.

- We enable others to generate their own world snippets by releasing our source code at https://github.com/Mawiszus/ World-GAN

\footnotetext{
${ }^{1}$ The smallest building block or tile a level is made of, e.g. sprites or voxels.
} 


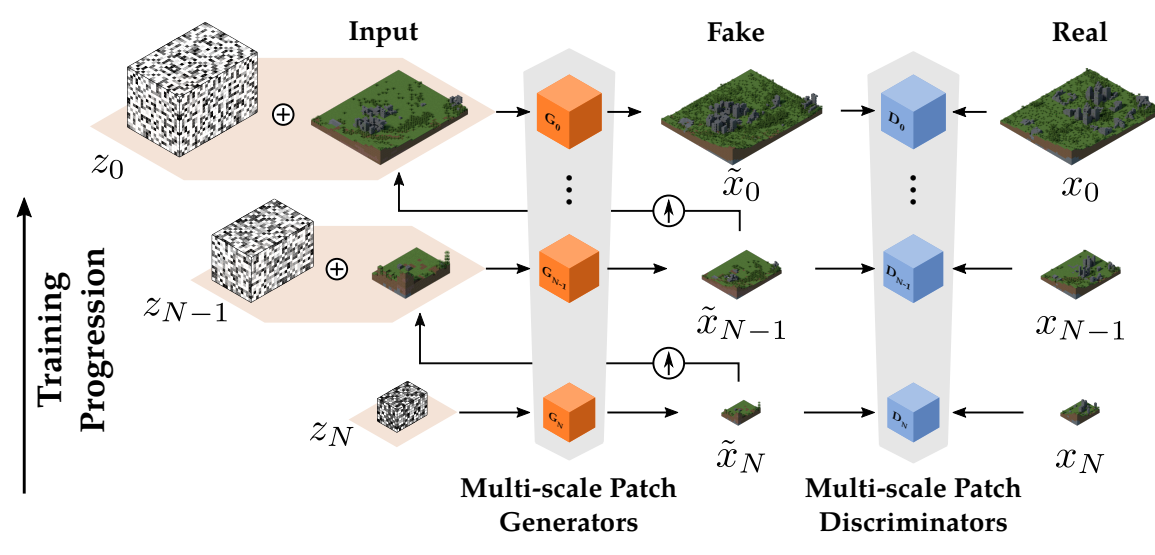

(a) Pipeline

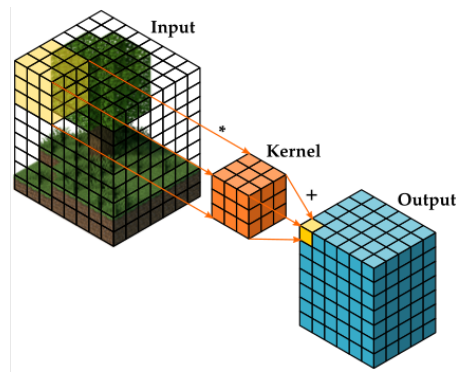

(b) 3D Convolution

Fig. 2: The World-GAN training pipeline. Similar to [10], different patch-based generators are trained at different scales to create locally convincing world snippets that the discriminators are trying to distinguish. While the original method uses one-hot encodings for their levels, we use dense representations from block2vec (Section III-E) that are mapped to functional levels after training. These representations make us independent of the number and types of blocks in a level. World-GAN uses 3D convolutions to process the 3D structures in the given world snippets.

\section{RELATED WORK}

The field of PCGML has seen many advances in recent years, due to the growing capabilities of Machine Learning algorithms. Besides classical methods like Markov Random Fields and GANs [11], its methods have been used for level generation in Candy Crush Saga and Super Mario Bros. (SMB) [5]. A recent approach framed PCG as a Reinforcement Learning (RL) problem and generated Zelda and Sokoban levels [12] using a Deep RL agent. Our method is inspired by our previous work TOAD-GAN [10], which extended SinGAN [13] to token-based games by using a hierarchical downsampling operation.

In contrast to these existing studies, we propose a method for 3D level generation in Minecraft that adapts the idea of token embeddings from Natural Language Processing (NLP) to overcome memory bottlenecks and manually-defined token hierarchies. Embeddings of game entities have not been used in PCG, but were posed as a future research direction in [14].

A game where PCG plays an essential role is Minecraft [7]. The complex 3D structures in this game pose a problem for PCGML methods. The AI Settlement Generation Challenge [15]-[17] was recently created to spur research in this direction. The submitted algorithms are generating villages in a given world and are evaluated using subjective measures (adaptability, functionality, narrative, aesthetics). The creators of the challenge mention data-driven approaches as a future direction of PCG in Minecraft which was one motivation for our work. One method to increase the diversity of the generated content was published by Green et al. [18], which generates floor plans using a constrained-growth algorithm and Cellular Automata.

Several simplified Minecraft-inspired simulators were proposed [19], [20] to study the creative space of 3D structures.
Grbic et al. [21] introduce the problem of open-ended procedural content generation in Minecraft. Sudhakaran et al. [22] use the Neural Cellular Automata architecture to produce a fixed structure in Minecraft given a seed or partial structure.

Yoon et al. [23] classify Minecraft villages into several themes (e.g. medieval, futurist, asian) but do not perform PCG. There have been experiments to generate Minecraft structures based on user-defined content [24], but the results were not satisfying. Our proposed World-GAN is one of the first practical PCGML applications for Minecraft.

\section{METHOD}

Our method builds upon several existing techniques which are briefly described in this section before we introduce WorldGAN and our block2vec algorithm.

\section{A. Generative Adversarial Networks}

World-GAN is based on the GAN [25] architecture. Given a dataset, these networks are able to generate new samples that are similar to the provided examples. They are trained by using two adversaries, a generator $G$ and a discriminator $D$. The generator is fed a random noise vector $z$ and produces an output $\tilde{x}$. Then, the discriminator is either given a real sample $x$ or the generated one and has to predict whether the sample is from the real dataset or not. By learning to fool the discriminator, the generator is gradually producing more and more samples that look as if they belong to the training distribution. One problem with this architecture is that it requires a lot of data. Otherwise, it is too easy for the discriminator to distinguish between real and fake samples and the generator is not able to improve its output.

\section{B. $\operatorname{Sin} G A N$}

SinGAN [13] enables the generation of images from only one example by using a fully-convolutional generator and 
discriminator architecture. Thus, the discriminator only sees one part of the sample and can more easily be fooled by the generator. Because the field of view in this architecture is limited, long-range correlations can only be modeled by introducing a cascade of generators and discriminators that operate at $N$ different scales. The samples for each scale are downsampled and the GANs are trained beginning from the smallest scale $N$

$$
\tilde{x}_{N}=G_{N}\left(z_{N}\right) .
$$

This scale defines the global structure of the generated sample, which will be refined in the subsequent scales. At scales $0 \leq n<N$, the output from the previous scale is upsampled $(\uparrow)$ and passed to the scale's generator after disturbing it with a noise map $z_{n} \sim \mathcal{N}\left(0, \sigma_{n}{ }^{2}\right)$. The variance of the noise determines the amount of detail that will be added at the current scale by the generator to produce

$$
\tilde{x}_{n}=\tilde{x}_{n+1} \uparrow+G_{n}\left(z_{n}+\tilde{x}_{n+1} \uparrow\right) .
$$

At each scale, the discriminator either receives a downsampled real sample $x_{n}$ or the output of the generator with equal probability. The gradient of the discrimination loss is then propagated to the discriminator and the generator, which creates the Minimax problem

$$
\min _{G_{n}} \max _{D_{n}} \mathcal{L}_{\text {adv }}\left(G_{n}, D_{n}\right)+\alpha \mathcal{L}_{\text {rec }}\left(G_{n}\right) .
$$

The loss $\mathcal{L}_{\text {adv }}$ is the widely-used Wasserstein GAN with Gradient Penalty (WGAN-GP) [26], [27] loss and $\mathcal{L}_{\text {rec }}$ is a reconstruction loss weighted by $\alpha$ which ensures that the GAN's latent space contains the real sample ${ }^{2}$ After training on one scale has converged, the parameters of the generator and discriminator are copied to the next scale as an initialization.

\section{TOAD-GAN}

As SinGAN is designed for modeling natural images, its application to token-based games requires some modifications. TOAD-GAN [10] introduces several changes to SinGAN's architecture. Small structures that consist of only a few or a single token would be missing at lower scales due to aliasing by the downsampling operation. The bilinear downsampling is thus replaced by a special downsampling operation that considers the importance of a token in comparison with its neighbors. The importance is determined using a hierarchy that is constructed by a heuristic which is motivated by the TFIDF metric from NLP. These extensions allow TOAD-GAN to be applied to SMB and several other 2D token-based games. However, the generation of $3 \mathrm{D}$ content requires some changes to the network architecture of TOAD-GAN. The jump from 2D to $3 \mathrm{D}$ means the size of samples will be significantly bigger and since TOAD-GAN is using one-hot encodings of tokens, the required GPU space grows substantially. This shortcoming is especially apparent in Minecraft, where the high number of tokens can drastically limit the volume that TOAD-GAN

\footnotetext{
${ }^{2}$ For a more detailed description see $[13]$ and $[10]$.
}

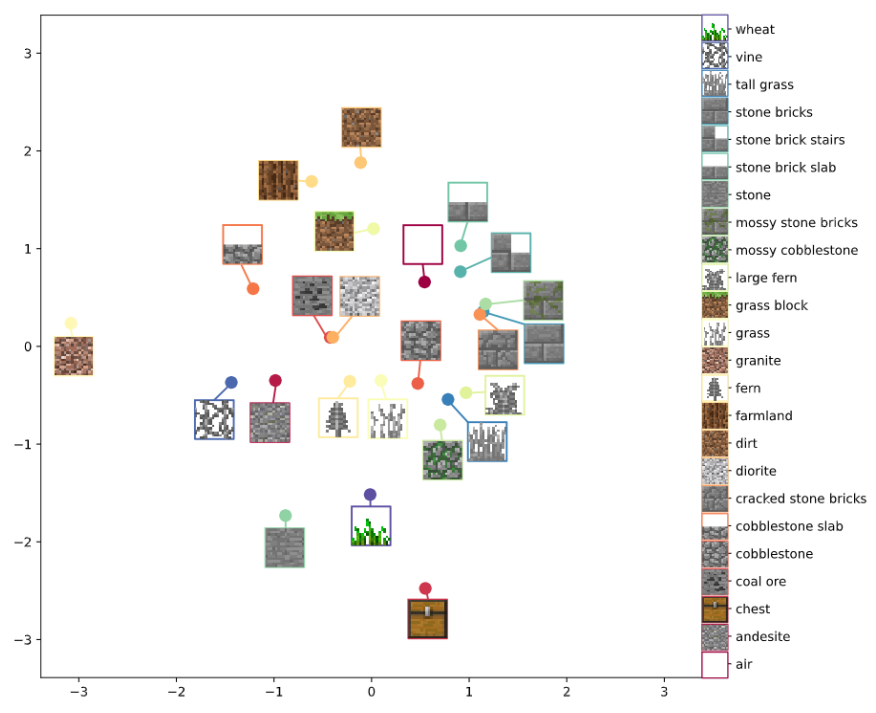

Fig. 3: Embeddings learned by block2vec of the ruins structure. The embeddings have 32 dimensions but are transformed to two dimensions for this visualization using the Minimum Distortion Embedding method [28].

is able to generate. To put this difference into perspective, a one-hot encoded tensor of the original SMB level 1-1 has a shape of $202 \times 16$ with 12 (out of 28 possible) different tokens. Taking only the actually present tokens into account, this results in 38,784 floating point numbers, which take up $0.16 \mathrm{MB}$. The village example by comparison has a shape of $121 \times 136 \times 33$ with 71 (out of $300+$ possible) different tokens, resulting in $38,556,408$ numbers that require 154.23 MB to store. If you do not preprocess the data so that only present tokens are taken into account, the difference becomes even more steep.

\section{World-GAN}

While the overall architecture of World-GAN in Fig. 2a is similar to TOAD-GAN, the 3D structure of Minecraft levels requires several modifications. The generator and discriminator now use 3D convolutional filters that can process the $k \times D \times$ $H \times W$ sized slices from the input level. Here, $k$ is the number of tokens in a level and $D, H$ and $W$ are the depth, height and width of the slice. Fig. $2 b$ shows a visualization of the 3D convolution operation.

Another difficulty is the number of tokens in Minecraft and their long-tailed distribution, i.e. some of the tokens only appear a few times in a given sample whereas others (such as air) take up half of the map. To make World-GAN independent of the number of tokens, we turn to a technique from NLP.

\section{E. block2vec}

Previous works on GANs [5], [10] for PCGML use a onehot encoding of each token in a level. The downsampling in TOAD-GAN's architecture requires a hierarchy of tokens to enable the generation of small structures at lower scales. This 
TABLE I: Structure coordinates of our example areas in DREHMAL:PRIMSRDIAL [29] (visualizations are shown in Fig. 4).

\begin{tabular}{lcccr}
\hline Structure & $\mathrm{x}$ & $\mathrm{y}$ & $\mathrm{z}$ & \multicolumn{1}{c}{ Volume } \\
\hline desert & {$[-3219,-3132]$} & {$[2628,2717]$} & {$[116,128]$} & 92916 \\
plains & {$[1082,1167]$} & {$[1110,1186]$} & {$[65,103]$} & 245480 \\
ruins & {$[1026,1077]$} & {$[1088,1152]$} & {$[63,73]$} & 32640 \\
beach & {$[606,695]$} & {$[-688,-629]$} & {$[39,64]$} & 131275 \\
swamp & {$[-2753,-2702]$} & {$[3242,3296]$} & {$[56,86]$} & 82620 \\
mine shaft & {$[24987,25029]$} & {$[-799,-754]$} & {$[20,38]$} & 34020 \\
village & {$[25165,25286]$} & {$[-770,-634]$} & {$[55,88]$} & 543048 \\
\hline
\end{tabular}

heuristic, based on term frequencies, has its limitations due to the large number of available tokens in Minecraft which is also constantly expanding in newer versions of the game. In NLP, this problem was solved by learning a dense fixed-size representation of words [1]. These embeddings are constructed by modeling the joint probability of a token and its context.

To train these block2vec token embeddings, we construct a dataset of blocks and their neighbors from the area of interest, i.e. we create block 2 vec embeddings for each new area we want to train. Some tokens such as air occur relatively often, which can lead to sub-optimal representations. Following [1], we mitigate this imbalance by sampling the tokens according to their occurrence probability

$$
P\left(b_{i}\right)=\sqrt{\frac{f\left(b_{i}\right)}{0.001}+1} \cdot \frac{0.001}{f\left(b_{i}\right)},
$$

where $f\left(b_{i}\right)$ is the frequency of the token $b_{i}$ in our given training sample.

We use a skip-gram model with two linear layers, i.e. predicting the context from the target token. Since our vocabulary is still relatively small in comparison to other NLP tasks, we do not have to employ negative sampling like Mikolov et al. [1]. This algorithm can be seen as a kind of matrix factorization [30] into an $m$-dimensional token representation and a token affinity matrix. Using a dimensionality reduction technique (MDE) [28], we can visualize our token embeddings (Fig. 3).

The generators and discriminators in World-GAN are given the levels in this representation space, i.e. the generator produces a $m \times D \times H \times W$ tensor that is fed to the discriminator. After training is complete, the generated levels can be turned into a valid Minecraft level by choosing the token whose embedding is the nearest-neighbor to the generator's output for each voxel. In contrast to the size calculated in Section III-C, the tensor for the village example now does not depend on the dimensionality of 71 in the token dimension but on the size of the token embedding. We choose 32 in our experiments, resulting in a tensor using $69.51 \mathrm{MB}$ instead. The size of 32 was empirically chosen, so it can be reduced even further by choosing a smaller representation dimensionality where appropriate.

In addition to reduced memory requirements, block $2 \mathrm{vec}$ also allows us to omit the definition of a hierarchy. The token hierarchy was proposed in [10] to enable the generation of
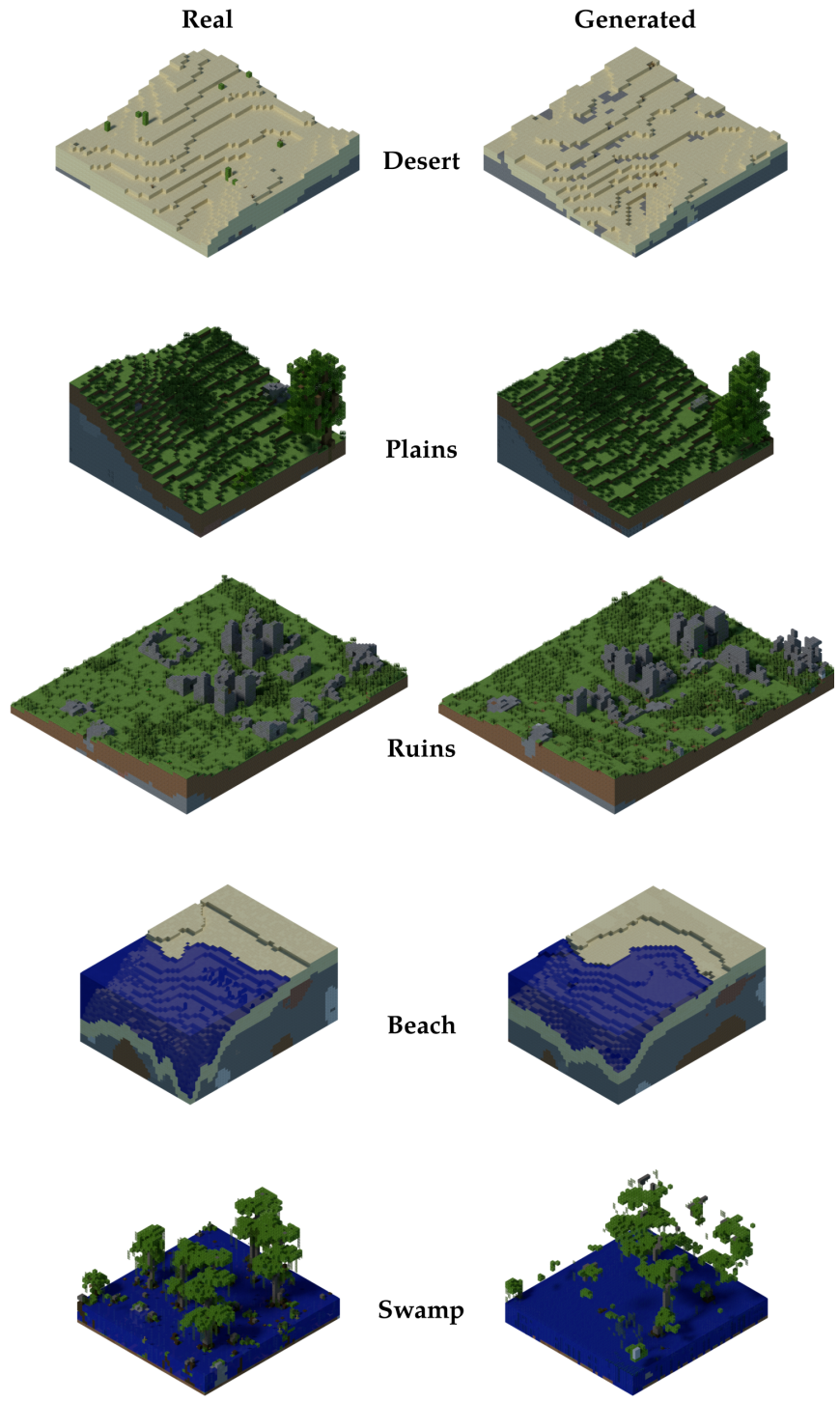

Swamp
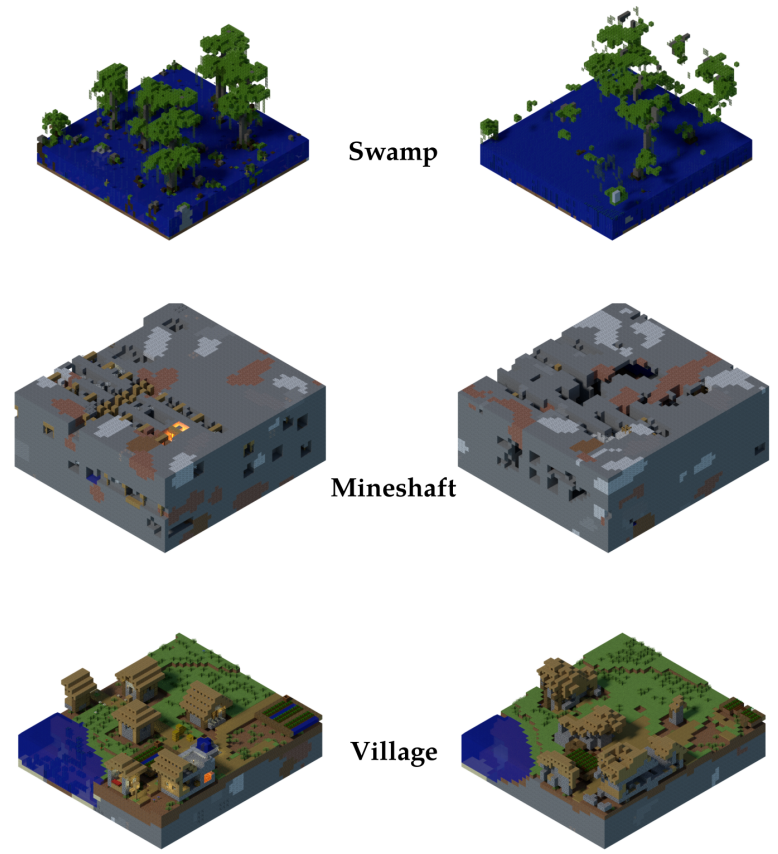

Fig. 4: Qualitative samples of our generated levels using block2vec. Using our learned representation space, WorldGAN is able to create convincing samples of different areas while still including meaningful variations. It excels at generating samples in which structures can be freely added and subtracted from, like ruins. 
small or rare tokens at lower scales. Making sure rare tokens are generated is especially important in video game levels, as gameplay relevant secrets or power-ups are usually rare and hidden on purpose. In our new embedding space, rare tokens are placed close to semantically similar more common tokens. For example, in the embeddings shown in Fig. 3 the stone brick stairs is a rare token, but its representation is close to one of the most common tokens, stone bricks. With this, a generator at higher scales can more easily learn to generate the rarer token even if the more common token was generated one scale below.

Finally, choosing a different mapping from internal representations to tokens allows us to change the style of the generated content after training, which we demonstrate in Section IV-D

\section{EXPERIMENTS}

We perform several experiments to evaluate the capabilities of our method, which we will describe in the following sections. After showing some qualitative samples for a range of different areas in Minecraft, we evaluate several metrics, such as the Tile Pattern KL-Divergence (TPKL-Div) and the Levenshtein Distance. We prove the effectiveness of our block2vec embedding, by comparing it to variants of TOADGAN which we extended to $3 \mathrm{D}$. We call these variants $T O A D$ $G A N 3 D$ and TOAD-GAN $3 D^{*}$, for a TOAD-GAN extended to $3 \mathrm{D}$ with and without hierarchica ${ }^{3}$ downsampling respectively. Additionally, we present one version of World-GAN that we train on embeddings of the token descriptions from a general purpose NLP model, called BERT [31]. Finally, we change the mapping from our representation space to tokens to showcase the possibility of editing World-GAN's output after training.

\section{A. Qualitative Examples}

Our goal is to generate areas for Minecraft that are similar to a user-defined world snippet but show a reasonable amount of variation. There is no restriction to what kind of blocks are in the snippet, therefore, any type of area (biomes, buildings, plants) can be used for training World-GAN. To demonstrate the broad applicability of our method we choose a variety of user-defined biomes, like a desert, plains and a beach in our experiments. We also want to investigate the capability of the method to create simple structures. For this we select samples with buildings or natural structures like ruins, swamp trees, a mine shaft and a village. For reproducibility, we extracted all of these samples from the handcrafted world "DREHMAL:PRIMSRDIAL" [29], which is available online. The coordinates in which the areas can be found are shown in Table II. The village and the mine shaft are drawn from areas of the original Minecraft World Generator. All other areas are taken directly from hand crafted biomes in [29].

Fig. 4 shows each of the different areas and one sample generated with our proposed World-GAN. We can see, that World-GAN is capable of reproducing a convincing sample of

\footnotetext{
${ }^{3}$ The hierarchy uses a token frequency based heuristic like in $[10]$.
}

the area while still showing some variability. Even structures can be generated to a certain degree, such as ruins and trees. However, as World-GAN is not optimized to make coherent, functioning structures, the details of generated houses, like the interior blocks, windows, doors and specific structure are not entirely correct. Still, the overall structure and style of all areas is captured well by our method.

For comparison, Fig. 5 shows a sample generated by using a simple hierarchy as in [10]. The rank of a block in the hierarchy is defined by the inverse of the sum of its occurrences, i.e. the blocks occurring most often like air and grass blocks are lower in the hierarchy, while blocks that are rare such as stairs and a chest are higher. We can see that TOAD-GAN 3D also works with its simple hierarchy. However, it is apparent that rarer blocks are not being generated as much. Especially in the area where grass and air blocks meet in the upper part of Fig. 5a, the rarer tall grass block is not generated.

\section{B. Quantitative Evaluation}

In this section, we describe three different metrics to evaluate our generated levels: block distribution histograms, the TPKL-Div and the Levenshtein distance.

1) Block Distribution: As shown in Section IV-A especially rare tokens are difficult for PCGML methods to model. By counting the occurrences and visualizing them as histograms we can empirically study whether our method is able to produce rare tokens given the limited number of examples. Fig. 6 shows how World-GAN compares to TOAD-GAN 3D under this metric. Both methods have varying success generating rare tokens. Usually, the discriminator will be more likely to label a sample with a rare token as fake. However, for World-GAN with block2vec the rare tokens share some similarities with other more common tokens and are not as easy to detect. This leads to more of them being generated, as can be seen in the bottom row of Fig. 6. Only the chest token is missing from World-GAN's output. As its embedding in Fig. 3 is also further away from the other tokens, we hypothesise that it might be too easy for the discriminator to detect compared to the other rare tokens. Developing different embeddings that generalise better between common and rare tokens is a direction for future work.

2) Tile Pattern KL-Divergence: Next, we evaluate how well the patterns in our generated content match the original sample based on the metrics used in [10]. The TPKL-Div [32] is the Kullback-Leibler Divergence of token patterns of size $n$ that occur in a level. We apply it to Minecraft by considering all $n \times n \times n$ patterns in our generated content

$$
D_{K L}(P \| Q)=\sum_{s \in \mathbb{N}^{n \times n \times n}} P(s) \log \frac{P(s)}{Q(s)},
$$

where $P(s)$ is the frequency of the pattern $s$ in the original level and $Q(s)$ is its frequency in the generated level. We choose patterns of size $5 \times 5 \times 5$ and $10 \times 10 \times 10$ and average their resulting TPKL-Divs. In preliminary experiments we found that using 4 scales of sizes $1.0,0.75,0.5$ and 0.25 


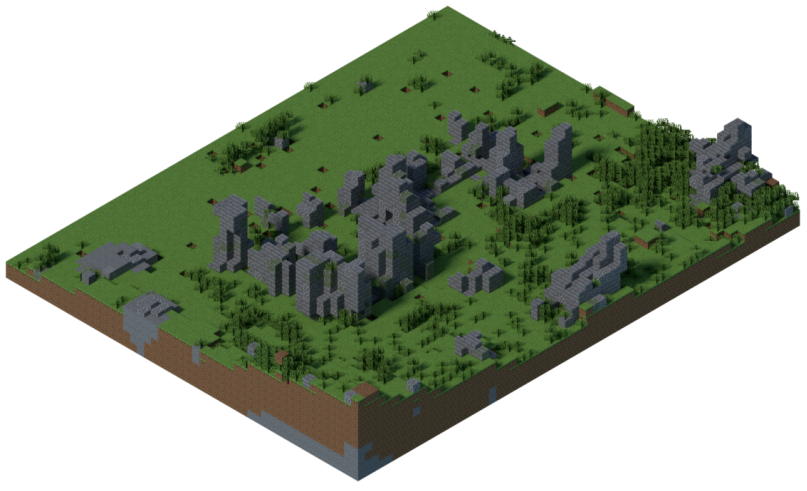

(a) TOAD-GAN 3D

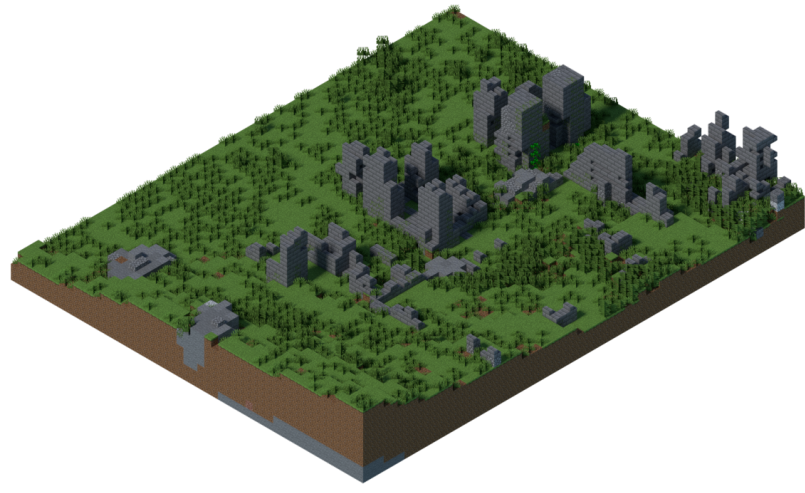

(b) World-GAN (block2vec)

Fig. 5: Qualitative comparison between content from TOAD-GAN 3D and our block2vec approach. (a) was generated with a simple hierarchy based on the method originally described in [10], and (b) is a sample from the same generator as in Fig. 4 The samples look very similar overall and both methods generate viable structures.

TABLE II: Average Tile-Pattern KL-Divergence between the real structure and 20 generated levels. A lower TPKL-Div implies that the patterns of the original level are matched better.

\begin{tabular}{lccc}
\hline & World-GAN & TOAD-GAN 3D & TOAD-GAN 3D* \\
\hline desert & $\mathbf{1 6 . 2 8}$ & 18.18 & 18.56 \\
plains & 23.05 & $\mathbf{2 2 . 7 9}$ & 22.84 \\
ruins & $\mathbf{1 6 . 2 0}$ & 16.35 & 16.51 \\
beach & 16.76 & $\mathbf{1 5 . 8 0}$ & 16.22 \\
swamp & 20.32 & $\mathbf{1 8 . 4 1}$ & 19.95 \\
mine shaft & 14.67 & 14.64 & $\mathbf{1 4 . 6 2}$ \\
village & 21.73 & 21.57 & $\mathbf{2 1 . 3 5}$ \\
\hline Average & 18.43 & $\mathbf{1 8 . 2 5}$ & 18.58 \\
\hline
\end{tabular}

in our generation process leads to the best results 4 . We use this configuration for all qualitative samples that are presented in the paper. The results in Table $\Pi$ II show that World-GAN with block $2 \mathrm{vec}$ is able to match the training patterns as well as the other variants while requiring less memory (compare Section III-D.

3) Levenshtein Distances: Finding an objective measure for the uniqueness of procedurally generated content is no easy task. The Levenshtein distance [33] is an established metric coming from information theory to measure the similarity of two discrete strings. It is defined as the minimum number of insertions, deletions and substitutions that are needed to transform one string into the other. The distance is bounded from above by the length of the longer string and is equal to zero iff. the two strings are equal. We can interpret slices of our generated levels as strings by concatenating the tokens at each position and assigning them a number. This allows us to compute the Levenshtein distance between all generated samples of an area, which lets us quantify their variability. The results are shown in Table III We find that content

${ }^{4}$ We evaluated World-GAN with scales $(1.0,0.5),(1.0,0.75,0.5)$ and $(1.0$, $0.75,0.5,0.25)$. The corresponding results are published with our source code.
TABLE III: Average Levenshtein distance between the generated levels. A larger distance implies a larger variability in the generated output.

\begin{tabular}{lccc}
\hline & World-GAN & TOAD-GAN 3D & TOAD-GAN 3D* \\
\hline desert & $\mathbf{3 2 5 1 . 3 7}$ & 1342.24 & 1305.19 \\
plains & $\mathbf{5 3 1 4 . 4 3}$ & 3895.96 & 4372.41 \\
ruins & 5073.49 & $\mathbf{5 8 0 8 . 9 7}$ & 5615.67 \\
beach & $\mathbf{1 5 6 2 3 . 5 7}$ & 12846.66 & 11743.38 \\
swamp & 7900.03 & $\mathbf{1 0 5 1 5 . 4 0}$ & 7292.57 \\
mine shaft & $\mathbf{9 6 9 1 . 6 9}$ & 6138.71 & 7764.37 \\
village & 5721.88 & 6452.61 & $\mathbf{6 6 7 9 . 6 9}$ \\
\hline Average & $\mathbf{7 5 1 0 . 9 2}$ & 6714.36 & 6396.18 \\
\hline
\end{tabular}

generated using block2vec has a higher variability on average. One explanation could be the generation of rare tokens, which are more frequent in World-GAN's output. This supports our findings in Section IV-B1

\section{Alternative Token Embeddings}

We evaluate the impact of the token embeddings on the generated output by comparing our block2vec approach to the canonical BERT [31] embeddings of the token descriptions. BERT is a widely used NLP model trained on many sample sentences of the English language and can therefore provide embeddings for any English sentence. For this experiment, we feed the token descriptions (e.g. mossy stone bricks) to a pretrained BERT model [34] and use the final layer's output as our token embedding. Fig. 7 shows a qualitative sample when training with these 768 dimensional embeddings. It is apparent that the patterns are not as closely modeled as with the block2vec embeddings. This could be attributed to the high dimensionality of the embeddings. However, despite not being trained on Minecraft, the general structure of stone ruins with grass around them is still visible. Since the embeddings are created using only their textual description, this experiment indicates a future research direction of grounding World-GAN in natural language. This is especially interesting regarding the recently introduced Chronicle Challenge [17]. 

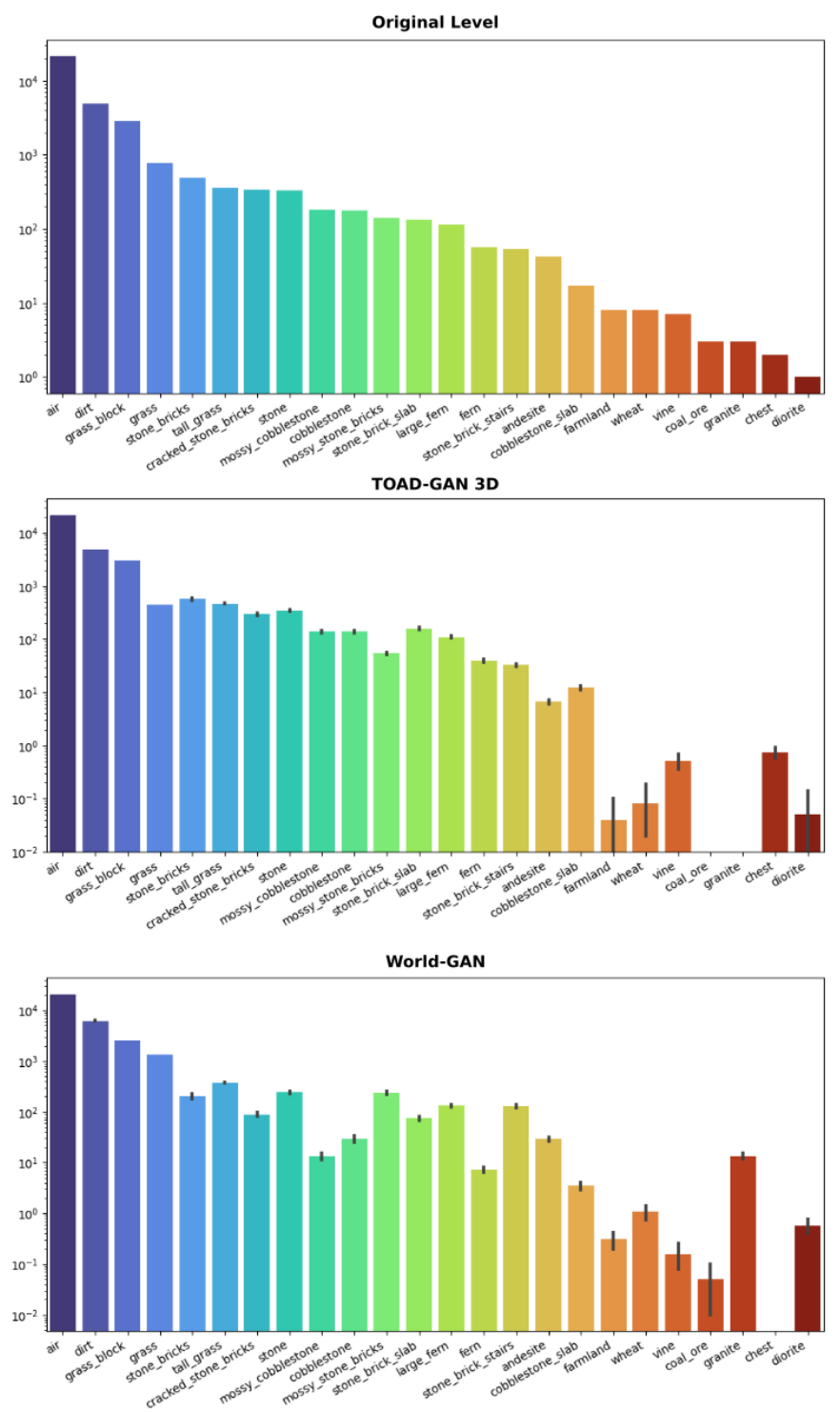

Fig. 6: Histograms depicting the block distributions for the ruins example, showing mean and variance over 100 generated samples. The y-axis is scaled logarithmically. While there are some differences in the token occurrence counts between our samples and the original, we capture the given distribution reasonably well and are also able to generate most rare tokens.

\section{Representation Editing}

Similar to TOAD-GAN, levels generated with World-GAN can be edited during generation. The editing capabilities discussed in [10] are all applicable to World-GAN as well. In this section, however, we want to highlight another possibility of editing the generated levels. As described in Section III-E. World-GAN is trained on a specific representation space. Since the generated samples also belong to this representation space, the transformation from that space back to the original tokens is crucial to the style of the generated level. By changing this interpretation of the latent space, we can change the style

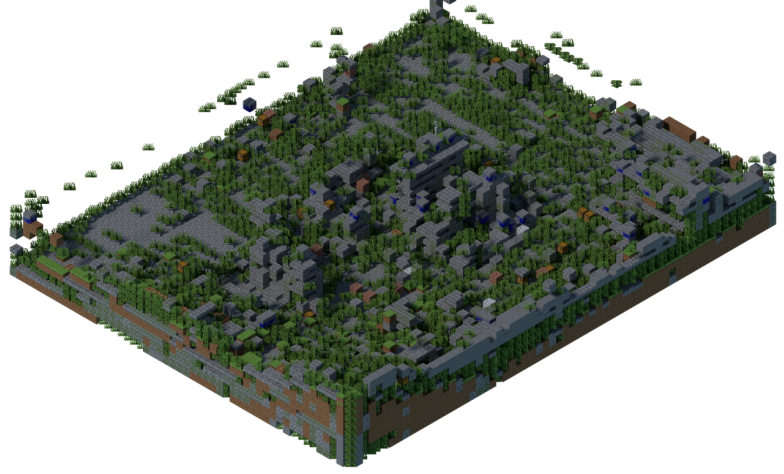

Fig. 7: Sample generated with a World-GAN trained using BERT embeddings of the token descriptions. Some artifacts such as floating tall grass are produced but the overall structure of the map is visible. This indicates that meaningful level generation within a learned word space is possible.

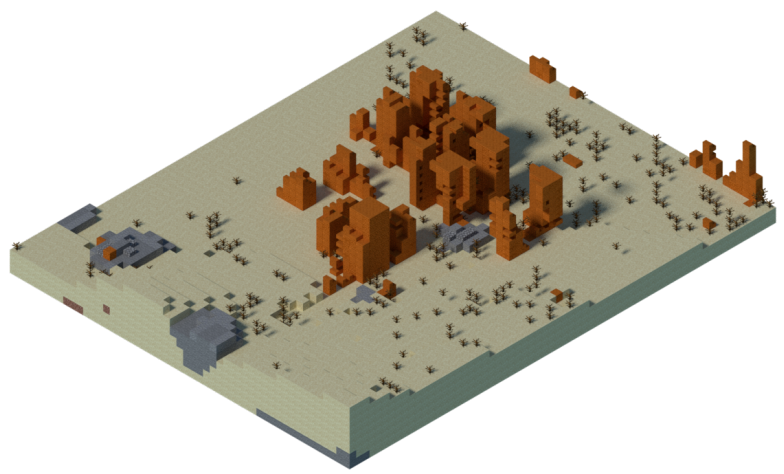

Fig. 8: An example of a level with edited representations. With block2vec, we can change the style of the generated structure (ruins) from one style (plains) to another (desert).

of the generated tokens without retraining the generator. This results in levels that have the same structure as the original training sample, but can use wildly different tokens. Fig. 8 shows an example of such a transfer. The World-GAN used in this example is the same generator used for the ruins examples in previous sections. For each token vector in the representation space we change the token it is mapped to, in order to fit the new style. In this example, we changed the blocks representing the ground, i.e. grass and dirt, to be interpreted as sand and the blocks representing the ruins, i.e. stone bricks, to variations of red sandstone. This method allows a designer to use the generator of one basic structure for many different styles.

\section{CONCLUSion ANd Future Work}

We introduce World-GAN, a method that enables datadriven PCGML in Minecraft. It is inspired by the TOADGAN [10] architecture, but is specifically extended to 3D by incorporating $3 \mathrm{D}$ convolutions and the application of dense token embeddings, which we call block2vec. We evaluated its generated content with respect to its pattern similarity to the original input, its variability and by how well it handles rare 
tokens in the input. Finally, we present an easy way to change the representations after training, which enables us to edit the style of a given level. Especially with the Minecraft Settlement Generation Challenge [16] in mind, a few research directions open up for future work. With the current method, semantic correctness of structures is not enforced, which can result in for example nonsensical houses. We want to investigate improving our method in order to better generate semantic structures by incorporating semantic rules into the generation process. Our method opens up several directions for PCGML in Minecraft, as we will publish our source code and are able to handle the most current Minecraft version.

\section{ACKNOWLEDGMENT}

This work has been supported by the Federal Ministry of Education and Research (BMBF), Germany, under the project LeibnizKILabor (grant no. 01DD20003), the Federal Ministry for Economic Affairs and Energy under the Wipano programme "NaturalAI" (03THW05K06), the Center for Digital Innovations (ZDIN) and the Deutsche Forschungsgemeinschaft (DFG) under Germany's Excellence Strategy within the Cluster of Excellence PhoenixD (EXC 2122).

\section{REFERENCES}

[1] T. Mikolov, K. Chen, G. Corrado, and J. Dean, "Efficient Estimation of Word Representations in Vector Space," arXiv:1301.3781 [cs], Sep. 2013.

[2] S. Snodgrass and S. Ontanón, "Generating maps using markov chains," in Ninth Artificial Intelligence and Interactive Digital Entertainment Conference, 2013

[3] J. Gutierrez and J. Schrum, "Generative Adversarial Network Rooms in Generative Graph Grammar Dungeons for The Legend of Zelda," in 2020 IEEE Congress on Evolutionary Computation (CEC). IEEE, 2020, pp. 1-8.

[4] A. Summerville, S. Snodgrass, M. Guzdial, C. Holmgård, A. K. Hoover, A. Isaksen, A. Nealen, and J. Togelius, "Procedural Content Generation via Machine Learning (PCGML)," IEEE Transactions on Games, vol. 10, no. 3, Sep. 2018.

[5] V. Volz, J. Schrum, J. Liu, S. M. Lucas, A. Smith, and S. Risi, "Evolving mario levels in the latent space of a deep convolutional generative adversarial network," in Proceedings of the Genetic and Evolutionary Computation Conference, 2018, pp. 221-228.

[6] E. Giacomello, P. L. Lanzi, and D. Loiacono, "Doom level generation using generative adversarial networks," in 2018 IEEE Games, Entertainment, Media Conference (GEM). IEEE, 2018, pp. 316-323.

[7] Mojang Studios, "Minecraft," 2009, version: 1.16.

[8] E. Haines, "Mineways," https://www.realtimerendering.com/erich/ minecraft/public/mineways/index.html version: 8.05.

[9] Bender Foundation, "Blender," https://www.blender.org/ version: 2.79.

[10] M. Awiszus, F. Schubert, and B. Rosenhahn, "Toad-gan: coherent style level generation from a single example," in Proceedings of the AAAI Conference on Artificial Intelligence and Interactive Digital Entertainment, vol. 16, no. 1, 2020, pp. 10-16.

[11] V. Volz, N. Justesen, S. Snodgrass, S. Asadi, S. Purmonen, C. Holmgård, J. Togelius, and S. Risi, "Capturing Local and Global Patterns in Procedural Content Generation via Machine Learning," in 2020 IEEE Conference on Games (CoG). IEEE, 2020, pp. 399-406.

[12] A. Khalifa, P. Bontrager, S. Earle, and J. Togelius, "Pcgrl: Procedural content generation via reinforcement learning," in Proceedings of the AAAI Conference on Artificial Intelligence and Interactive Digital Entertainment, no. $1,2020$.

[13] T. R. Shaham, T. Dekel, and T. Michaeli, "Singan: Learning a generative model from a single natural image," in Proceedings of the IEEE International Conference on Computer Vision, 2019, pp. 4570-4580.

[14] N. Y. Khameneh and M. Guzdial, "Entity Embedding as Game Representation," in Proceedings of the Second Workshop on Experimental AI in Games - EXAG '20, Oct. 2020.
[15] C. Salge, M. C. Green, R. Canaan, F. Skwarski, R. Fritsch, A. Brightmoore, S. Ye, C. Cao, and J. Togelius, "The AI Settlement Generation Challenge in Minecraft: First Year Report," KI - Künstliche Intelligenz, vol. 34, no. 1, Mar. 2020.

[16] C. Salge, M. C. Green, R. Canaan, and J. Togelius, "Generative design in minecraft (GDMC): Settlement generation competition," in Proceedings of the 13th International Conference on the Foundations of Digital Games. Malmö Sweden: ACM, Aug. 2018.

[17] C. Salge, C. Guckelsberger, M. C. Green, R. Canaan, and J. Togelius, "Generative Design in Minecraft: Chronicle Challenge," in 10th International Conference on Computational Creativity, ICCC 2019. Association for Computational Creativity (ACC), 2019, pp. 311-315.

[18] M. C. Green, C. Salge, and J. Togelius, "Organic building generation in minecraft," in Proceedings of the 14th International Conference on the Foundations of Digital Games. San Luis Obispo California USA: ACM, Aug. 2019.

[19] L. B. Soros, J. K. Pugh, and K. O. Stanley, "Voxelbuild: A minecraftinspired domain for experiments in evolutionary creativity," in Proceedings of the Genetic and Evolutionary Computation Conference Companion. Berlin Germany: ACM, Jul. 2017.

[20] C. Patrascu and S. Risi, "Artefacts: Minecraft meets collaborative interactive evolution," in 2016 IEEE Conference on Computational Intelligence and Games (CIG). Santorini, Greece: IEEE, Sep. 2016.

[21] D. Grbic, R. B. Palm, E. Najarro, B. Glanois, and S. Risi, "EvoCraft: A New Challenge for Open-Endedness," in Applications of Evolutionary Computation: 24th International Conference, EvoApplications 2021, Held as Part of EvoStar 2021, Virtual Event, April 7-9, 2021, Proceedings, vol. 12694. Springer Nature, 2021, p. 325.

[22] S. Sudhakaran, D. Grbic, S. Li, A. Katona, E. Najarro, C. Glanois, and S. Risi, "Growing 3D Artefacts and Functional Machines with Neural Cellular Automata," arXiv:2103.08737 [cs], Mar. 2021

[23] E. Yoon, E. Andersen, B. Hariharan, and R. A. Knepper, "Design mining for minecraft architecture," in AIIDE, 2018.

[24] M. T., "Minecraft-gan-city-generator," https://github.com/BluShine/ Minecraft-GAN-City-Generator 2020.

[25] I. Goodfellow, J. Pouget-Abadie, M. Mirza, B. Xu, D. Warde-Farley, S. Ozair, A. Courville, and Y. Bengio, "Generative adversarial nets," in Advances in Neural Information Processing Systems, 2014.

[26] M. Arjovsky, S. Chintala, and L. Bottou, "Wasserstein generative adversarial networks," in International Conference on Machine Learning, 2017, pp. 214-223.

[27] I. Gulrajani, F. Ahmed, M. Arjovsky, V. Dumoulin, and A. C. Courville, "Improved Training of Wasserstein GANs," in Advances in neural information processing systems, 2017, pp. 5767-5777.

[28] A. Agrawal, A. Ali, and S. Boyd, "Minimum-distortion embedding," arXiv, 2021

[29] "Drehmal:primordial," https://www.planetminecraft.com/project/ drehmal-v2-prim-rdial-12k-x-12k-survival-adventure-map/ accessed: 2021-03-26.

[30] O. Levy and Y. Goldberg, "Neural Word Embedding as Implicit Matrix Factorization," Advances in neural information processing systems, 2014.

[31] J. Devlin, M.-W. Chang, K. Lee, and K. Toutanova, "BERT: Pre-training of Deep Bidirectional Transformers for Language Understanding," in Proceedings of the 2019 Conference of the North American Chapter of the Association for Computational Linguistics: Human Language Technologies, Volume 1 (Long and Short Papers), 2019, pp. 4171-4186.

[32] S. M. Lucas and V. Volz, "Tile Pattern KL-Divergence for Analysing and Evolving Game Levels," Proceedings of the Genetic and Evolutionary Computation Conference, Jul. 2019.

[33] V. I. Levenshtein, "Binary Codes Capable of Correcting Deletions, Insertions and Reversals," Soviet Physics Doklady, vol. 10, p. 707, Feb. 1966.

[34] T. Wolf, L. Debut, V. Sanh, J. Chaumond, C. Delangue, A. Moi, P. Cistac, T. Rault, R. Louf, M. Funtowicz, J. Davison, S. Shleifer, P. von Platen, C. Ma, Y. Jernite, J. Plu, C. Xu, T. L. Scao, S. Gugger, M. Drame, Q. Lhoest, and A. M. Rush, "Transformers: State-ofthe-art natural language processing," in Proceedings of the 2020 Conference on Empirical Methods in Natural Language Processing: System Demonstrations. Online: Association for Computational Linguistics, Oct. 2020, pp. 38-45. [Online]. Available: https: //www.aclweb.org/anthology/2020.emnlp-demos.6 